\title{
El origen de la universidad Incluyente en México: Su evolución y retos
}

The origin of the Inclusive University in Mexico: Its evolution and challenges

\author{
Araiza Santillán Marisa Aideé ${ }^{1 凶}$, Palafox Bolívar Marisol Priscila ${ }^{1}$, Torres López Octavio , Álvarez \\ Loya Liliana , Méndez González José Emilio ${ }^{1}$
}

${ }^{1}$ Facultad de Universidad Autónoma de Chihuahua, Facultad de Contaduría y Administración. Calle $6{ }^{\text {a }}$ y ave 21 poniente sector poniente s/n Delicias, Chihuahua

Autor para correspondencia: maraiza28@yahoo.com.mx

Recibido: $18 / 04 / 2020$

Aceptado: 24/05/2020

\section{RESUMEN}

Desde el desarrollo de los tiempos, la educación ha ido evolucionando, ya que esto ha traído la inclusión de alumnos con diversas variantes en su desarrollo, y con esto podemos ver que en México, ha sido una continua inclusión dentro de las formas de educar. En la que hemos visto un crecimiento de matrícula muy adecuado y lo que nos indica un desarrollo continuo del proceso de crecimiento de la UACH. En el proceso de realización de esta investigación pudimos ver como se ha ido incrementando la matrícula y la aceptación de los alumnos con discapacidad, los cuales han ido ganando terreno en el ámbito educativo. Al final concluimos, que a pesar de todo, y pese a todos los altibajos que ha sufrido nuestra economía y sociedad, se acaba de dar un vuelco a ver las necesidades de este sector tan dañado, y tan frágil de nuestra sociedad, esos pequeños-grandes guerreros, que no importando su condición, luchan por seguir en esta tierra, por ganar un espacio y que quieren ser tratados, como lo que son Unos Seres Especiales, aquellos, que por naturaleza, por accidente o por enfermedad, dejaron de desarrollar algún sentido, o bien lo desarrollan poco, pero que al entrar al habito educativo pueden lograr sus metas y sus ideales.

Palabras claves: Discapacidad, Educación, Evolución, Alumnos, Maestros.

\begin{abstract}
Since the development of time, education has evolved, since this has brought the inclusion of students with various variants in their development, and with this we can see that in Mexico, it has been a continuous inclusion within the ways of educating. In which we have seen a very adequate enrollment growth and which indicates a continuous development of the UACH growth process. In the process of carrying out this research, we could see how enrollment and acceptance of students with disabilities have increased, which have been gaining ground in the educational field. In the end, we conclude that despite everything, and despite all the ups and downs that our economy and society have suffered, we have just turned around to see the needs of this very damaged and fragile sector of our society, those little- great warriors, who regardless of their condition, fight to continue on this earth, to gain a space and who want to be treated, like what Some Special Beings are, those who, by nature, by accident or illness, stopped developing any sense, or they develop little, but that by entering the educational habit they can achieve their goals and ideals.
\end{abstract}


Keywords: Disability, Education, Evolution, Students, Teachers.

\section{INTRODUCCION}

En México la transición de los métodos de educación antiguos y actuales se llevó a cabo de una forma intermitente pero siempre con el firme objetivo de integrar a toda la población a una educación global, no enfocándose únicamente a la población considerada apta para el aprendizaje. En las últimas décadas se generaron notables cambios en la evolución sistemática de la educación, primero a nivel básico o elemental, y después a nivel profesional, lo cual se refleja en el crecimiento interno de nuestra población a pesar de las diferencias internas del país.

El avance más importante se dio, cuando se desarrolló el plan para la inclusión de todas las personas a la educación, por medio de "un México incluyente" el cual, creado por la Lic. Josefina Vázquez Mota, como propuesta de campaña 2012, y la cual fue respaldada por el Presidente de la Republica en ese momento, Enrique Peña Nieto, lo cual dio un gran impulso en 2013, al apoyo a las escuelas para poder contar con todas las instalaciones adecuadas para la aceptación e ingreso de alumnos con discapacidad. La Educación Especial en México tiene una larga historia, sin embargo ésta no existe realmente como política educativa y como sistema de atención a la población, sino hasta después de 1970 con la creación de la Dirección General de Educación Especial (DGEE) y la ampliación de servicios hacia las entidades federativas. Es importante hacer mención de este tipo de educación, (especial), ya que es parte de la evolución educativa que ha sido representativa en nuestro país, al contarse con ahora escuelas que no solo imparten conocimientos a personas con capacidades normales, sino diferentes.
Los modelos de atención han cambiado cada década, de ahí que en 42 años de existencia se hayan experimentado cuatro, en los que sin evaluar los resultados de uno, se encima otro, lo que ha desarrollado una práctica profesional en la que se combinan unos y otros sin que asuman precisión alguna. Por esta razón, es por la que los modelos educativos, van creando una vertiente de progreso y mejora para el desarrollo de las personas que se incluyen dentro de este grupo, con discapacidad.

Dentro de este estudio también, enfocaremos parte de este análisis en la información de los recursos que se asignan de manera anual al presupuesto de la educación, y como ha sido vulnerable el que se ha otorgado a las personas con discapacidades, las cuales, tienen los mismos derechos y las mismas oportunidades que los todos los miembros de esta sociedad.

El desarrollo de la educación especial es consecuencia de la aplicación de una política de Estado que al inicio de la década de los setenta se denomina "Educación para todos" y que va a determinar el reconocimiento de la educación especial como una acción del Estado a través de la cual se atiende a los niños "atípicos", como eran llamados en esa época.

Mondragon (2011) mencionó que "el sistema educativo en México fue por muchos años víctima de la inercia política e institucional del antiguo poder hegemónico, creo con grandes costos al sindicato más grande de América Latina y en la actualidad necesita de una reforma educativa que ponga al país a la altura de la nueva realidad mundial".

Con base a esta observación que realiza Mondragón, es importante señalar que ciertamente nuestro país ha ido observando 
grandes cambios de manera significativa que desembocan en la mejora continua de la población con oportunidad de desarrollase, ya no únicamente en el área profesional, sino educativa, trayendo como consecuencia una población con mayor capacidad de desarrollo y de incremento en sus niveles de educación.
En específico, en el estado de Chihuahua, el nivel educativo profesional cambió considerablemente en los últimos 10 años, la matrícula profesional dentro de las Universidades ha tenido los siguientes cambios:

Tabla 1. Incremento de la educación en diferentes modalidades.

\begin{tabular}{|l|l|}
\hline \multicolumn{1}{|c|}{ PERIODO } & \multicolumn{1}{c|}{ INCREMENTO } \\
\hline & -En licenciatura un 32.80 \%. \\
Del año 2004 al 2009 & -En el Sistema Abierto y Virtual un 121.41\%. -En \\
(UACH, 2011). & nivel Técnico un 140.48\%. \\
& -A nivel Post Grado un 31.54\%. \\
\hline Del 2009 al 1015: & -A nivel Licenciatura 22\%. \\
$(\mathrm{UACH}, 2015)$. & -A nivel Post Grado 31\%. \\
\hline
\end{tabular}

Fuente: (UACH, 2011; 2015).

En la tabla anterior se observó el incrementado de la matricula a todos los niveles de educación, y en especial en el área de preparación profesional a nivel licenciatura y post grado.

Como se ha descrito brevemente, la educación ha sufrido muchos y grandes cambios durante su historia por lo que es importante que en este trabajo se analicen a profundidad los cambios por lo que ha pasado, principalmente en el tema sobre la inclusión de personas con discapacidad a la educación global.

\section{MATERIALES Y METODOS}

Para generar la información se realizó una investigación bibliográfica exhaustiva partiendo de la educación prehispánica hasta la actualidad, una vez teniendo la información se analizó y se plasmaron los resultados por apartados.

\section{RESULTADOS}

A partir de la revisión de diferentes medios bibliográficos sobre la evolución de la educación desde tiempos prehispánicos a los contemporáneos se obtuvieron los siguientes resultados que fueron divididos por secciones:

Prehispánica. La educación en la familia, el trabajo, la religión y la guerra, de acuerdo con algunas evidencias, tuvo características comunes en las culturas mesoamericanas, y este es el caso de los templos-escuelas, instituciones encargadas de transmitir a los niños y jóvenes, bajo una rígida disciplina, conocimientos religiosos y militares.

Pese a las distintas referencias en los ordenamientos jurídicos internos los hijos pequeños de los nobles eran cuidados y vigilados en su conducta por servidores, mientras que los niños del pueblo aprendían y heredaban el oficio de sus padres y les ayudaban en las actividades cotidianas. Las niñas aprendían las tareas de la madre, generalmente relacionadas con el trabajo del hogar o el comercio (Larroyo, 1979).

Durante la Colonia. Existieron diversos tipos de enseñanza: evangelizadora, adiestramiento en artes y oficios, femenina, religiosa $\mathrm{y}$ 
universitaria. Las actividades educativas fueron asumidas por diversas órdenes religiosas. A partir de la segunda mitad del siglo XVI, los jesuitas llegarían a ejercer una gran influencia intelectual en la sociedad novohispana.

Durante las primeras décadas de la Conquista, los franciscanos ofrecieron educación en internados conventuales a los hijos de señores y principales mexicas, con el doble objetivo de enseñarles la lectura y la escritura del español y evangelizar a los indígenas. Esos jóvenes mexicas participaron eficazmente en la labor catequizadora. Los internados adoptaron elementos de los templos-escuelas que guardaban semejanzas con la educación conventual, como el rigor de la vida de los internos, la retórica ceremonial y la formación moral (Flores González, 2012).

En el periodo Post-Independiente. Una vez consumada la independencia de México, se abrió un periodo de intensas luchas entre liberales y conservadores, dentro de un proceso histórico de construcción del Estado nacional. En cuatro décadas el país perdió la mitad de su territorio, sufrió la intervención armada de Estados Unidos de Norteamérica y de Francia y estuvo gobernado durante algunos años por un noble austríaco, impuesto por la alianza que establecieron los sectores conservadores con Napoleón III.

Los liberales y los conservadores coincidían en que la educación era fundamental, pero sus profundas diferencias políticoideológicas y los conflictos con el exterior dificultaron la construcción del Estado y, con ello, la definición de políticas educativas. No obstante, si se compara con el periodo colonial, la educación en este periodo tuvo avances, en especial la primaria que se extendió a gran parte del país (Staples, 1992).
En el Porfiriato. El poder y los recursos económicos tendieron a centralizarse a costa de las autonomías locales y estatales. La antigua base municipal de la educación fue erosionándose por la supresión de las alcabalas. La falta de recursos locales y nacionales dificultó el desarrollo de un sistema nacional y unitario de educación pública (Martinez J., 1999, p. 26).

La expansión del sistema educativo. Entre 1920 y 1950 el sistema educativo mexicano creció en forma constante pero moderada. Durante esos años, la enseñanza primaria se concentraba principalmente en el medio urbano y los niveles superiores tenían un carácter restringido. Ese patrón de crecimiento acabó a mediados de la década cincuenta, dando lugar a un gran ciclo expansivo de treinta años que concluyó en los años ochenta.

La presidencia de Adolfo López Mateos (1958-1964). Consideró prioritario ampliar oportunidades educativas, apoyar la educación normal y la capacitación para el trabajo. Logró más educación y capacitación para la población, concentrándose en el centro de la Republica. Acumulación de matrícula 58.2\% (INEGI, 1990).

\section{La presidencia de Gustavo Díaz Ordaz}

(1964-1970). Las propuestas educativas fueron semejantes a las de su antecesor, sin embargo, el ritmo de crecimiento del sistema disminuyó, debido a la situación social que se vivía en ese momento en nuestro país. Acumulación de matrícula 42.2\% (INEGI, 1990). 
La presidencia de Luis Echeverría Álvarez, 1970-1976. Propició la expansión y diversificación de los servicios educativos, la multiplicación de las instituciones en todo el país y su crecimiento interno, la reforma a los planes y programas de primaria y secundaria, la edición de nuevos libros de texto gratuito y la promulgación de nuevas leyes en materia educativa y de patrimonio cultural.

Creó el Consejo Nacional de Fomento Educativo (CONAFE), el Centro de Estudios de Métodos y Procedimientos Avanzados de la Educación (CEMPAE), el Consejo Nacional de Ciencia y Tecnología (CONACYT), Departamento de Investigaciones Educativas del CINVESTAVIPN y del Centro de Investigaciones Superiores del Instituto Nacional de Antropología e Historia (INAH) y el Sistema de Primaria Intensiva para Adultos. Se promulgó la Ley Federal sobre Monumentos y Zonas Arqueológicas, Artísticas e Históricas, la cual afirmó la propiedad nacional del patrimonio histórico y artístico, así como su protección y exploración (Liwerant, 2003).

La presidencia de José López Portillo, 19761982. El gobierno de López Portillo elaboró el Plan Nacional de Educación (PNE) que consistió en un diagnóstico y en un conjunto de programas y objetivos. En 1978 se declaró prioritaria la educación preescolar y se puso en marcha el Programa de Educación para Todos, se impulsó la educación terminal, se reguló la educación superior mediante la planeación creando la Universidad Pedagógica Nacional (UPN). En este sexenio adquirieron relevancia las preocupaciones sobre la calidad y la atención al estudiantado (Liwerant, 2003).

\section{La presidencia de Miguel de la Madrid en} adelante 1982 en adelante. El gasto del gobierno, en especial el destinado a las áreas sociales sufrió una drástica caída, la crisis afectó la demanda escolar en los sectores de menores ingresos y aumentó las probabilidades de abandono y reprobación escolar. El sistema educativo mexicano interrumpió las tendencias expansivas que lo habían caracterizado desde décadas anteriores (Liwerant, 2003), quedando a un lado la inclusión de la educación para personas con discapacidades, ya que en nuestro país no existían los medios ni las herramientas para poder incluirlos en el contexto de la educación.

Problemática de la educación a personas discapacitadas. Históricamente, las personas con alguna o con múltiples discapacidades habían quedado restringidas al hogar o, cuando mucho, a instituciones segregadas que han venido llamándose colectivamente de "Educación Especial". Ese paradigma se basa en el convencimiento de que estas personas no pueden educarse y que constituyen una carga para el sistema de enseñanza regular. De hecho, hay una frecuente renuncia por parte de las escuelas regulares a matricular a los estudiantes con discapacidad o, cuando la matricula se concreta, de expulsarlos porque resulta más difícil educarlos.

La práctica de separar a los estudiantes con discapacidad puede entrañar su mayor marginación social, situación en la que se encuentran generalmente las personas con discapacidad, y con ello afianzar la discriminación. En cambio, se ha demostrado que la educación inclusiva, derecho de todos y todas, al promover la convivencia con la diferencia y lo plural, impulsa la superación de estereotipos, de prejuicios y, por ende, de la discriminación. La inclusión de los estudiantes con discapacidad en las escuelas regulares desde la más temprana edad, por otro lado, confiere importantes ventajas psicológicas, las cuales atiende mucho más las necesidades intelectuales, 
sociales y emocionales mediante una interacción regular con un grupo diverso de estudiantes y es una de las mejores maneras de combatir estereotipos y promover la conciencia sobre las capacidades de las personas con deficiencia.

Evolución de la forma de enseñanza. En relación a lo anterior veremos una breve descripción de la evolución que ha tenido nuestro país, en relación a la educación, y como se ha reflejado el desarrollo intelectual de los maestros en torno a la educación de los niños:

-La pedagogía tradicional comienza a gestarse en el siglo XVII con las escuelas públicas en Europa y América Latina, con el éxito de las revoluciones buscadas en la doctrina política y social del liberalismo, buscando implementar la educación traída de España. En esta etapa se concede a la escuela el valor de ser la institución social para todas las capas sociales, para la construcción de la nación y reconocimiento moral y social, esta escuela tradicional, adquiere carácter de pedagógica, dándole un énfasis al desarrollo educativo de los niños de la colonia.

-El proceso evolutivo de la educación llega a considerar a la escuela como el medio ideológico y cultural con propósitos de formar a los jóvenes, enseñarle los valores y la ética, así como educarlos en las conductas de la comunidad, en tanto al maestro se le considera el centro del proceso de enseñanza, trasmisor de información y sujeto del proceso de enseñanza, piensa y transmite los conocimientos con poco margen para que el alumno elabore y trabaje mentalmente, de tal forma que se inicia el proceso de desarrollo mental y social de los alumnos.

-En un punto de la educación el maestro exige memorización, que el alumno narre y exponga exactamente la información que se le solicita el contenido de la enseñanza es llamada enciclopedista e intelectualista. Los temas son aislados, sin relacionarse a las experiencias del alumno. El estudiante desarrolla un pensamiento empírico con carácter clasificador y se orienta por las cualidades externas del objeto y sus propiedades aisladas

-La educación del aprendizaje va dirigida al resultado, sin análisis y razonamiento $\mathrm{El}$ profesor es expositivo, ofrece una cantidad de información para ser memorizada por el alumno, aquí se establece la idea de que "la letra con sangre entra". La relación del alumno-profesor se basa en el predominio del profesor autoritario $\mathrm{y}$ al alumno es pasivo, tal vez por temor o por la autoridad que este representa para el alumno.

-Los roles que debía asumir el alumno eran determinados por su género o aspecto físico más que por su capacidad o habilidad, claro que dentro de este contexto, los alumnos con discapacidades no entraban en el grupo de los enseñables.

-La vestimenta de los maestros debía ser completamente formal: Traje y corbata para los hombres y vestido largo con zapatillas para las mujeres.

-Los castigos físicos eran muy comunes en la educación tradicional en México. Se pretendía convencer al alumno de la necesidad de comportarse debidamente, imperaban los valores y el respeto a sus autoridades.

Dichos castigos incluían jalón de orejas, hincar al alumno, golpear con una tabla, dar bofetadas o nalgadas, arrojar el borrador al alumno entre otros castigos severos.

-Ahora la pedagogía tradicional no es la tendencia más adecuada para resolver la tarea que tiene la enseñanza, al contrario, ahora se basa en el respeto mutuo y la libertad de expresión, en donde algunos de los casos, se han propasado en una vestimenta inadecuada, en un mal comportamiento del alumno con el maestro y de una vestimenta, en muchos de los casos inadecuada.-Diversos docentes y pedagogos han 
propuesto reformas al modelo tradicional buscando realizar mejoras al proceso enseñanzaaprendizaje, y dentro de este contexto, se ha incluido la lucha por un México Incluyente, donde no únicamente las bases educativas se enfoquen a las personas que con sus habilidades y posibilidades físicas, mentales y emocionales, puedan desarrollar su educación, desde la básica, hasta una educación profesional completa.
Estadísticas en materia de personas con discapacidades. Direccionando la atención en específico a las personas con discapacidades en México, se tienen registros del censo 2015, publicado en el 2016, en relación a los índices de discapacidad detectados, teniendo la siguiente información:

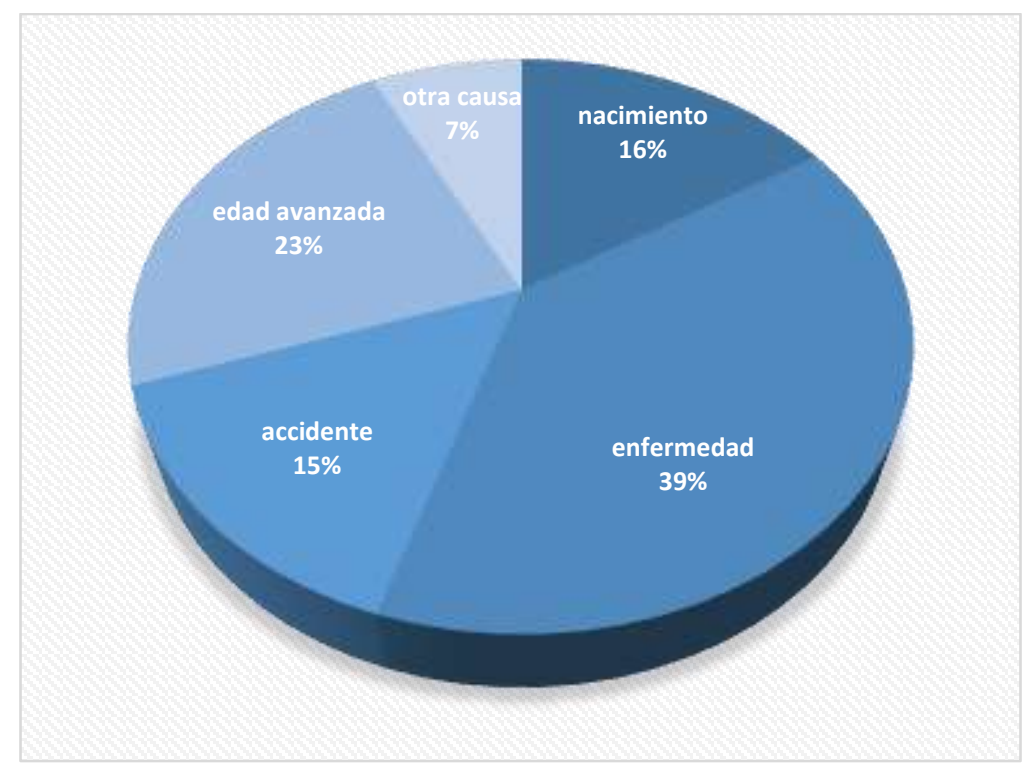

Figura 1. Causales de Discapacidad en México.

Fuente: Instituto Nacional de Estadísticas (INEGI) 2015

Los causales de discapacidad se disparan en lo referente a enfermedad, siguiéndolo a la edad avanzada, y siendo de gran importancia los que se han provocado por accidente $\mathrm{y}$ por nacimiento, motivo por el cual, se han implementado esta Ley del México Incluyente. (Impreso Politica, Nov 2012), en la cual se establece una serie de acciones que generan el desarrollo y la inclusión de las personas con discapacidad en México y otorga facultades a la administración pública federal para la elaboración de políticas que generan su desarrollo y con ello mejorar su calidad de vida.
Con la entrada en vigor de esta ley, el Ejecutivo federal y el Congreso resuelven una asignatura pendiente que desde hace más de una década se tenía con las personas con discapacidad.

Coalición México por los Derechos de las Personas con Discapacidad (COAMEX) indicó en 2012 que los legisladores fueron "muy generosos" en la construcción del marco jurídico, pensando en el bien de las personas con discapacidad, y sólo queda esperar lo mismo para el resto de los presupuestos. Por tal motivo, a partir de esta fecha se estableció que durante el ejercicio 2013, el área de educación para 
personas Especiales, se verá beneficiada, ya que siento este un rubro tan importante en el área social de nuestro país, y se le dio un gran impulso, en el desarrollo de las aulas y de los espacios especiales para la inclusión de alumnos con discapacidades.

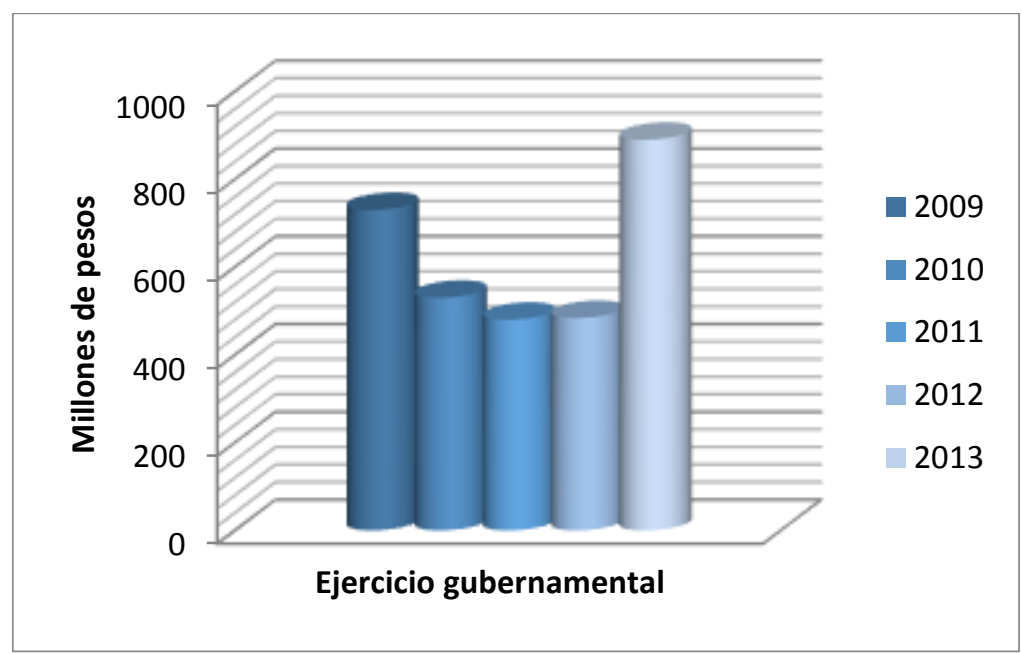

Figura 2. Presupuesto Gubernamental para Educación a personas con discapacidades. Fuente: Revista radis abril del 2010 y transparecia presupuestaria de Gob. Federal 2012.

El gráfico anterior nos muestra el decremento considerable que existió en nuestro país durante el 2009 al 2012, y el impulso que se le dió apartir del ejercicio 2013, esto muestra que fue de suma importancia haber realizado una Ley para la proteccion de las personas con discapacidad ya que ha generado mayor apoyo a este sector tan fragil y desprotejido de nuestra sociedad.

\section{DISCUSIÓN}

Existe un gran debate acerca de si la educación para jóvenes con alguna discapacidad debe darse de manera discriminada o de modo inclusivo, sin embargo no se trata simplemente decir hagamos una escuela inclusiva, porque en la realidad las instituciones de educación públicas especialmente en América latina sin tener inclusión ya presentan muchas dificultades, en sus métodos de enseñanza los cuales no están acorde de los cambios de la tecnología, ignorando las necesidades de los jóvenes actuales, son escuelas masivas donde las diferencias individuales son pasadas por alto, donde se le da mayor importancia a la cantidad que a la calidad, teniendo en cuenta esto la inclusión no es la mejor alternativa, pues si en la actualidad la escuela no es un lugar donde se respete la diferencia, teniendo niños especiales se incrementaría más esta dificultad creándole más problemas a la comunidad educativa.

Uno de los temas álgidos cuando se habla de educación es el tema del afecto, esta discusión cobra más importancia a la hora de hablar de educación especial puesto que en este tipo de educación el alumno requiere mayor compromiso de parte del docente pues la discapacidad le exige al profesor más estrategias, mayor creatividad para hacer que sus alumnos comprendan y aprendan y una mayor tolerancia frente a las diferencias de sus alumnos, además cuando un profesor enseña a alumnos con discapacidad, se movilizan sus 
recursos afectivos, salen a la luz sus prejuicios acerca de la discapacidad.

En ese sentido Vergara (2000) dice que dentro de este contexto tan importante como lo son los maestros en nuestra comunidad estudiantil, y como se integran también los niños con discapacidades son demandantes de una gran cantidad de afecto especialmente al conocer las historias de ellos podemos identificar muchas experiencias en las que los padres por no aceptar las discapacidades de los hijos los abandonan o les maltratan son niños que crecen en situaciones carentes de afecto y de muestras de ternura, por tanto al llegar al aula escolar esta demanda de afecto recae en la profesora quien aunque no debería ser la primera fuente de afecto de los niños en ocasiones se convierte en la única que lo proporciona y ahí se vuelve a la pregunta inicial ¿favorece el proceso educativo de los niños especiales que los profesoras de los niños sean afectuosas? Los niños con profesores afectuosos demuestran una mejor disposición y motivación en el aula, presentan mejores relaciones con sus compañeros y sus actitudes son más positivas, los profesores afectuosos se preocupan más por tener un contacto individual con cada niño (aun en grupos grandes) identificando la manera en que el niño debe ser tratado y conociendo la forma adecuada de reforzar positivamente a su alumno, al realizar contacto físico con el niño el profesor le transmite una seguridad mayor y favorece la formación de una autoestima adecuada, cuando el niño ve en su profesor a una persona cercana puede confiar en el cuándo en el y tendrán a quien acudir en caso de necesitarlo, por ejemplo, cuando el niño se sienta solo e imposibilitado para realizar sus funciones básicas, el tener la confianza de buscar a su profesor para pedir apoyo en cualquier caso. Un profesor afectuoso es más tolerante a las dificultades que presente el alumno, a los comportamientos desadaptados que presente ocasionados por su discapacidad o por las carencias afectivas a que ha sido sometido, el afecto se puede demostrar con lenguaje verbal o no verbal dentro del aula de clase, contacto visual afectuoso, una caricia, un abrazo, una palabra de felicitaciones o de aliento, pedir a sus compañeros que lo aplauda cuando ha realizado una acción positiva. De igual manera un profesor afectuoso no deja de lados las normas y los límites, el profesor afectuoso debe manejar un equilibrio para determinar cuándo debe demostrar al alumno que su comportamiento fue inadecuado y la manera en que debe corregir su conducta. De igual manera debe evitar involucrase demasiado pues esto puede hacer que se afecte con las dificultades del niño lo que le impedirá tomar decisiones adecuadas y objetivas, por eso debe tener muy presente cuál es su rol como maestro sin exceder sus funciones para de esta manera dar un trato afectuoso a todos sus alumnos.

Y como dice (Bergeri, 2008) "La terapia familiar sistémica basa su trabajo en estas ideas y plantea que la generación de un cambio en el contexto siempre va a influir a los miembros del mismo y que, cuando un individuo genera un cambio, éste influye en el sistema total.” En lo cual estamos de acuerdo, debido a todo lo anteriormente mencionado, sabemos que la educación en este ámbito es de la familia completa.

También en (Castorina, Dic. 2011) nos dice que "Otro tanto puede decirse del Síndrome de Déficit Atencional: desde el punto de vista de la escuela socio histórica, los fenómenos psíquicos no pueden ser considerados como inmutables y dados para siempre, hay un desenvolvimiento histórico de esos fenómenos, una relación de dependencia esencial con respecto a la vida y a la actividad. 
En esta perspectiva teórica, una buena parte de los trastornos del aprendizaje ya no son comprendidos como fenómenos naturales puramente individuales orgánicos; se explican por la apropiación parcial de la actividad depositada en las producciones humanas, materiales o intelectuales" y con esto confirmando lo antes dicho por la Dra. Bergeri, en los párrafos anteriores.

\section{CONCLUSIÓN}

Para concluir con este tema que es de gran importancia $y$ ha servido para el conocimiento de este sector tan vulnerable, es necesario mencionar lo siguiente: Dar trabajo y estudio a las personas en situación de discapacidad es cada día un reto más importante al que se enfrentan las instituciones educativas y las empresas. Cada día las personas con discapacidad comprenden que ellos pueden tener una vida autónoma y productiva por lo que se interesan no solo en capacitarse a nivel técnico profesional, sino que también requieren un espacio laboral para aprovechar esas habilidades. Ser discapacitado no necesariamente quiere decir que sea dependiente económicamente de otros, por lo que se necesita apertura de las empresas para contratarlos, sin embargo algunas empresas se escudan para no tener empleados con discapacidad en algunos mitos tales como que las personas discapacitadas se ausentan mucho de sus trabajos por cuestiones de enfermedad o terapias y también porque consideran demasiado trabajo realizar condiciones óptimas en la planta física para ellos. Por ello es indispensable que empresarios reciban información de las ventajas que tiene contratar a personas con discapacidades, que el gobierno otorgue beneficios a éstas empresas para incentivar la contratación, ya que las personas discapacitadas en los entornos laborales o educativos tienden a ser más responsables y a trabajar con una mejor disposición.

Solo resta concluir, que a pesar de todo, y pese a todos los altibajos que ha sufrido nuestra economía y sociedad, se acaba de dar un vuelco a ver las necesidades de este sector tan dañado, y tan frágil de nuestra sociedad, esos pequeñosgrandes guerreros, que no importando su condición, luchan por seguir en esta tierra, por ganar un espacio y que quieren ser tratados, como lo que son Unos Seres Especiales, aquellos, que por naturaleza, por accidente o por enfermedad, dejaron de desarrollar algún sentido, o bien lo desarrollan poco, pero que a la par, se les desarrollo un sentido de grandeza inigualable, son únicos guerreros, luchadores, reyes dentro de esta tierra de caníbales, pero suaves y dulces como un cordero en su lecho, tenemos que seguir apoyando que se instalan cada vez más lugares aptos para ellos, lugares donde se pueda establecer un área de distracción, sin el temor a que no tengan donde bajar la silla de ruedas, o poner las muletas, áreas libres de humo, libres de delincuencia y con seguridad civil y moral, vamos juntos por un México incluyente, adelante.

\section{LITERATURA CITADA}

Larroyo, F. (1979). Historia Comparada de la Educacion en Mexico. Mexico: Porrua.

Castorina, J. (Dic. 2011). Las psicologias en la educacion especial. Educacion, lenguaje y sociedad, 15.

Liwerant, J. B. (2003). Las Ciencias Sociales, Universidad y Sociedad. Mexico: UNAM.

Bergeri, T. Z. (2008). Aportes a la perspectiva sistemica y la terapia familiar al trabajo en educacion especial. psicologia y educacion, 76.

Flores Gonzalez, J. I. (2012, abril 28). www.odiseo.com.mx/articulos/bosquejo- 
historico-de-la-educacion-formal-del-

cuerpo-en-Mexic-de-los-pueblos-

prehispanicos-al-siglo-XIX. (U. IPN,

Ed.) Retrieved diciembre 30, 2011, from

www.odiseo.com.mx

Guillen, R. J. (n.d.).

Impreso Politica. (Nov 2012). Solicitan mas

gasto para las personas con

discapacidad. Impreso Politica.

INEGI. (1990). Crecimiento porcentual de la matricula por sexenios 1952-1988.

Mexico: INEGI.

INEGI. (2010). Indice de causales de dispacacidad. mexico, DF: gobierno de la republica.
Martinez J., A. (1999). La Educacion:fuerza productivay catalizador del desarrollo. Mexico: UAM-Xochimilco.

Mondragon, J. C. (2011). Avances y retos de la educacion en Mexico.

Staples, A. (1992). Historia de la Vida cotidiana en Mexico:Bienes y Vivencias del siglo XIX (2a edicion ed., Vol. 4). (FCE, Ed.) Mexico, Mexico: Fondo de Cultura Economica.

UACH. (2011, febrero 11). Planeacion. Retrieved diciembre 19, 2009, from www.uach.mx/planeacion/2011/02/11/a genda-estadistica-2009.pdf

UNICEF. (2009). Educacion en Mexico.

Vergara, L. J. (2000). Inteligencia Emocional. Argentina: Goleman.

Copyright (c) 2020 Araiza Santillán Marisa Aideé, Palafox Bolivar Marisol Priscila, Torres López Octavio, Álvarez L oya Liliana, Méndez González José Em ilio

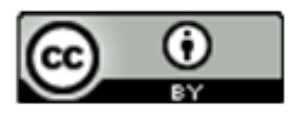

Este texto está protegido por una licencia licencia CreativeCommons 4.0.

Usted es libre para Compartir — copiar y redistribuir el material en cualquier medio o formato-y Adaptar el documento — remezclar, transformar y crear a partir del material- para cualquier propósito, incluso para fines com erciales, siempre que cumpla la condición de:

Atribución: Usted debe dar crédito a la obra original de manera adecuada, proporcionar un enlace a la licencia, e in dicar si se han realizado cambios. Puede hacerlo en cualquier forma razonable, pero no de forma tal que sugiera que tiene el apoyo del licenciante o lo recibe por el uso que hace de la obra.

Resumendelicencia - Textocompletodelalicencia 